\title{
A Banach space dichotomy theorem for quotients of subspaces
}

\author{
by \\ VALENTin FEREnCZI (Paris)
}

\begin{abstract}
A Banach space $X$ with a Schauder basis is defined to have the restricted quotient hereditarily indecomposable property if $X / Y$ is hereditarily indecomposable for any infinite-codimensional subspace $Y$ with a successive finite-dimensional decomposition on the basis of $X$. The following dichotomy theorem is proved: any infinite-dimensional Banach space contains a quotient of a subspace which either has an unconditional basis, or has the restricted quotient hereditarily indecomposable property.
\end{abstract}

1. Introduction. In 2002, W. T. Gowers published his famous Ramsey theorem for block-subspaces in a Banach space [8]. If $X$ is a Banach space with a Schauder basis, block-vectors in $X$ are non-zero vectors with finite support relative to the basis, and block-sequences are infinite sequences of block-vectors with successive supports; block-subspaces are subspaces generated by block-sequences.

If $Y$ is a block-subspace of $X$, Gowers' game in $Y$ is the infinite game where Player 1 plays block-subspaces $Y_{n}$ of $Y$, and Player 2 plays normalized block-vectors $y_{n}$ in $Y_{n}$.

If $\Delta=\left(\delta_{n}\right)_{n \in \mathbb{N}}$ is a sequence of reals, $\Delta>0$ means that $\delta_{n}>0$ for all $n \in \mathbb{N}$. For a set $A$ of normalized block-sequences, and any $\Delta=\left(\delta_{n}\right)_{n \in \mathbb{N}}>0$, let $A_{\Delta}$ be the set of normalized block-sequences $\left(y_{n}\right)_{n \in \mathbb{N}}$ such that there exists $\left(x_{n}\right)_{n \in \mathbb{N}}$ in $A$ with $\left\|x_{n}-y_{n}\right\| \leq \delta_{n}$ for all $n \in \mathbb{N}$.

Theorem 1 (Gowers' Ramsey theorem). Let $A$ be a set of normalized block-sequences which is analytic as a subset of $X^{\omega}$ with the product of the norm topology on $X$. Assume that every block-subspace of $X$ contains a block-sequence in A. Let $\Delta>0$. Then there exists a block-subspace $Y$ of $X$ such that Player 2 has a winning strategy in Gowers' game in $Y$ for producing a sequence $\left(y_{n}\right)_{n \in \mathbb{N}}$ in $A_{\Delta}$.

2000 Mathematics Subject Classification: 46B03, 03E15.

Key words and phrases: Gowers' dichotomy theorem, unconditional basis, hereditarily indecomposable, quotient of subspace, combinatorial forcing. 
The most important consequence of the Ramsey theorem of Gowers is the so-called dichotomy theorem for Banach spaces. A Banach space $X$ is said to be decomposable if it is a direct (topological) sum of two infinitedimensional closed subspaces. An infinite-dimensional space is hereditarily indecomposable (or $H I$ ) when it has no decomposable subspace. A Schauder basis $\left(e_{n}\right)_{n \in \mathbb{N}}$ of $X$ is unconditional if there exists $C \geq 1$ such that for all $\sum_{i \in \mathbb{N}} \lambda_{i} e_{i}$ in $X$, and all $\left(\varepsilon_{i}\right)_{i \in \mathbb{N}} \in\{-1,1\}^{\mathbb{N}},\left\|\sum_{i \in \mathbb{N}} \varepsilon_{i} \lambda_{i} e_{i}\right\| \leq C\left\|\sum_{i \in \mathbb{N}} \lambda_{i} e_{i}\right\|$.

Theorem 2 (Gowers' dichotomy theorem). Every infinite-dimensional Banach space contains a subspace $Y$ which has one of the following two properties, which are both possible, and mutually exclusive:

(i) $Y$ has an unconditional basis,

(ii) $Y$ is hereditarily indecomposable.

These properties are even exclusive in the sense that if a subspace satisfies (i) (resp. (ii)), then no further subspace satisfies (ii) (resp. (i)). Indeed, if a Banach space $X$ is hereditarily indecomposable, then so is any subspace of $X$; and if $X$ has an unconditional basis, then every block-subspace of $X$ has an unconditional basis, and so any subspace of $X$ has a further subspace with an unconditional basis.

1.1. HI spaces and their quotient spaces. From now on, spaces and subspaces are supposed to be infinite-dimensional and closed unless specified otherwise. For two subspaces $Y$ and $Z$ of a space $X$, a convenient notion of angle was used by B. Maurey to give a simple proof of Gowers' dichotomy theorem [12]: let

$$
a(Y, Z)=\inf _{y \in Y, z \in Z, y \neq z} \frac{\|y-z\|}{\|y+z\|} .
$$

It is in particular clear that $a(Y, Z) \neq 0$ if and only if $Y+Z$ is a topological direct sum in $X$, and therefore $X$ is hereditarily indecomposable if and only if $a(Y, Z)=0$ for any subspaces $Y, Z$ of $X$. On the other hand, a basic sequence $\left(e_{i}\right)_{i \in \mathbb{N}}$ is $C$-unconditional if $a\left(\left[e_{i}: i \in I\right],\left[e_{i}: i \in J\right]\right) \geq 1 / C$ for every partition $\{I, J\}$ of $\mathbb{N}$, where $\left[e_{i}: i \in I\right]$ denotes the closed linear space generated by $\left(e_{i}\right)_{i \in I}$.

We also note that it was proved in [9] that hereditarily indecomposable spaces are never isomorphic to proper subspaces.

While classical spaces, such as $c_{0}$ and $\ell_{p}, 1 \leq p<\infty$, or $L_{p}, 1<p<\infty$, have unconditional bases, the first known example of a HI space was given by Gowers and Maurey in 1993, [9]. Gowers-Maurey's space $X_{\mathrm{GM}}$ is actually quotient hereditarily indecomposable (or $Q H I$ ), that is, no quotient of a subspace of $X_{\mathrm{GM}}$ is decomposable, or equivalently, every infinite-dimensional quotient space of $X_{\mathrm{GM}}$ is $\mathrm{HI}$ [6]; as $X_{\mathrm{GM}}$ is reflexive, it follows that $X_{\mathrm{GM}}^{*}$ is also quotient hereditarily indecomposable, and in particular also hereditarily 
indecomposable. In [6], an example $X$ was also provided which is $\mathrm{HI}$ and not QHI. This example is defined as the "push-out" $\left(X_{1} \oplus X_{2}\right) /\{(y,-y): y \in Y\}$ of two specific Gowers-Maurey's type spaces $X_{1}$ and $X_{2}$ with respect to a "common" subspace $Y$. It is therefore still very close to being QHI, in the sense that it is saturated with QHI subspaces, and the natural quotient space of $X$ which is decomposable is a direct sum of two HI spaces. This led the author to conjecture that any quotient of a HI space should contain a HI or even QHI subspace, or that the dual of any reflexive HI space should contain a HI subspace.

This, however, turned out to be completely false. Examples of HI spaces were built with quotients which are very far from being HI. Using methods based on the definition of some notion of HI interpolation of Banach spaces, S. Argyros and V. Felouzis constructed a HI space with some quotient space isomorphic to $c_{0}$ (resp. $\left.\ell_{p}, 1<p<\infty\right)$ [2]. S. Argyros and A. Tolias used deep constructions, based on what is now known as the "extension method" [1], to prove that any separable Banach space which does not contain a copy of $\ell_{1}$ is isomorphic to the quotient space of some separable HI space [4]; and to construct a reflexive Banach space $X$ which is HI but whose dual is saturated with unconditional basic sequences [5], therefore any quotient space of $X$ has a further quotient with an unconditional basis. These results shatter all hopes of general results on preserving the HI property when passing to quotient spaces, or to the dual. We refer to [3], [4], and [11] for more details about these examples and hereditarily indecomposable spaces in general, as well as about other examples, and also to the recent work [1] which contains a comprehensive introduction to the previous examples.

S. Argyros asked whether there exists a reflexive HI Banach space $X$ such that no subspace of $X$ has a HI dual. This would show that the HI structure is in general not inherited by duals, not even in a very weak sense. None of the HI examples constructed so far seem to answer that question (for more about this, we refer to the remarks and questions section at the end of this paper).

Our main result is somewhat related to the question of Argyros. Its starting point is the observation that the situation becomes more pleasant again when one looks at quotients of subspaces (or QS-spaces) of a given Banach space. First note that the features of the QHI property with respect to quotients of subspaces are quite similar to the ones of the HI property with respect to subspaces. Indeed, this property obviously passes to further QS-spaces. We also have the following result.

Proposition 3. If $X$ is hereditarily indecomposable, then $X$ is isomorphic to no proper quotient of a subspace of itself. 
Proof. Assume $X$ is $\mathrm{HI}$ and $\alpha$ is an isomorphism from $X$ onto $Y / Z$ for some $Z \subset Y \subset X$. We may assume that $\operatorname{dim} Z=\infty$. Then by properties of HI spaces [12], the quotient map $\pi: Y \rightarrow Y / Z$ is strictly singular. The map $T=\alpha^{-1} \pi$ is an onto map whose Fredholm index $i(T)$ (defined as $\operatorname{dim}(\operatorname{Ker} T)-\operatorname{dim}(X / T Y)$ when this expression has a meaning) is $+\infty$. By continuity of the index ([10, Proposition 2.c.9]), we deduce that $i\left(T-\varepsilon i_{Y X}\right)=+\infty$ for some small enough $\varepsilon>0$. On the other hand, $T$ is strictly singular, therefore, by [10, Proposition 2.c.10], $i\left(T-\varepsilon i_{Y X}\right)=$ $i\left(-\varepsilon i_{Y X}\right) \leq 0$.

The unconditional property also has some type of heredity for quotients of subspaces. T. Odell proved that if $X$ has a shrinking finite-dimensional unconditional decomposition, then every normalized weakly null sequence in a quotient of $X$ has an unconditional subsequence [13], and therefore every QS-space of $X$ contains an unconditional basic sequence.

It is therefore tempting to look for some general dichotomy result for quotients of subspaces involving the QHI property on the one hand and some unconditionality property on the other.

1.2. Angles between quotients of subspaces. To motivate our definitions and results, we take a closer look at Gowers-Maurey's sequence space $X_{\mathrm{GM}}$. To prove that $X_{\mathrm{GM}}$ is HI, Gowers and Maurey build, for arbitrarily large $k \in \mathbb{N}$, successive biorthonormal sequences $\left(y_{i}\right)_{i \leq k}$ and $\left(y_{i}^{*}\right)_{i \leq k}$ of "special" pairs of vectors and functionals such that

$$
\left\|\sum_{i \leq k} y_{i}^{*}\right\| \simeq \sqrt{\log (k)}
$$

while

$$
\left\|\sum_{i \leq k}(-1)^{i} y_{i}\right\| \simeq k / \log (k) .
$$

Up to a perturbation, the terms $\left(y_{i}\right)_{i \leq k}$ may be taken in arbitrary subspaces of $X_{\mathrm{GM}}$. Therefore, given $Y, Z \subset X_{\mathrm{GM}}$, by taking the even terms close enough to $Y$ and the odd terms close enough to $Z$, we may find vectors $y$ almost in $Y$ and $z$ almost in $Z$, and functionals $y^{*}$ and $z^{*}$ with disjoint supports, such that $\|y-z\| \simeq k / \log (k)$ while

$$
\|y+z\| \geq \frac{\left(y^{*}+z^{*}\right)(y+z)}{\left\|y^{*}+z^{*}\right\|} \simeq k / \sqrt{\log (k)}
$$

It follows that $Y+Z$ is never a direct sum.

The proof in [6] that $X_{\mathrm{GM}}$ is QHI is based on the fact that one can actually choose $y^{*}$ and $z^{*}$ close enough to $W^{\perp}$ for any $W$ which is an infinitecodimensional subspace of $Y$ and of $Z$. It follows easily that $X_{\mathrm{GM}}$ is quotient hereditarily indecomposable. From the proof it is clear that one can even 
pick $y^{*}$ close enough to $V^{\perp}$ and $z^{*}$ close enough to $W^{\perp}$ for any infinitecodimensional subspaces $V$ of $Y$ and $W$ of $Z$. The point here is that each term of the sequences of "special" vectors (resp. functionals) must be taken in some set $A_{n}$ (resp. $A_{n}^{*}$ ) which is asymptotic, i.e. intersects any subspace of $X_{\mathrm{GM}}$ (resp. $X_{\mathrm{GM}}^{*}$ ), and depends on the previous terms of the sequences, but the subspace from which we pick it may be chosen arbitrarily.

For a Banach space $X$, and a subspace $Y_{*}$ of $X^{*}$, denote by $\|\cdot\|_{Y_{*}}$ the seminorm defined on $X$ by $\|x\|_{Y_{*}}=\sup _{y^{*} \in Y_{*},\left\|y^{*}\right\| \leq 1} y^{*}(x)$, and by $Y_{*}^{\perp}$ the orthogonal of $Y_{*}$ in $X$. When $Y_{*}=Y^{\perp}$ for some $Y \subset X,\|\cdot\|_{Y_{*}}$ is the quotient norm on $X / Y$.

A $Q S$-pair is some $\left(Y_{*}, Y\right) \subset X^{*} \times X$ such that $Y_{*}^{\perp} \subset Y$. It may be associated to the QS-space $Y / Y_{*}^{\perp}$. The natural notion of inclusion between QS-pairs

$$
\left(Z_{*}, Z\right) \subset\left(Y_{*}, Y\right) \Leftrightarrow\left(Z_{*} \subset Y_{*}\right) \wedge(Z \subset Y)
$$

corresponds to taking quotients of subspaces of the associated QS-spaces. Indeed, if $\left(Z_{*}, Z\right) \subset\left(Y_{*}, Y\right)$, then $Z / Z_{*}^{\perp} \simeq\left(Z / Y_{*}^{\perp}\right) /\left(Z_{*}^{\perp} / Y_{*}^{\perp}\right)$. An infinitedimensional QS-pair is a QS-pair whose associated QS-space is infinitedimensional. We define the angle $A\left(\left(Y_{*}, Y\right),\left(Z_{*}, Z\right)\right)$ between two QS-pairs by

$$
A\left(\left(Y_{*}, Y\right),\left(Z_{*}, Z\right)\right)=\inf _{y \neq z, y^{*} \neq z^{*}, y^{*}(z)=z^{*}(y)=0} \frac{\|y-z\|\left\|y^{*}-z^{*}\right\|}{\left|y^{*}(y)-z^{*}(z)\right|},
$$

where the infimum is taken over $y \in Y, z \in Z, y^{*} \in Y_{*}, z^{*} \in Z_{*}$.

Note that if we let $W_{*}=Y_{*}=Z_{*}$, then we obtain

$$
A\left(\left(W_{*}, Y\right),\left(W_{*}, Z\right)\right) \geq \inf _{y \neq z,\left\|y^{*}-z^{*}\right\|=1} \frac{\|y-z\|}{\left|\left(y^{*}-z^{*}\right)(y+z)\right|} \geq \inf _{y \neq z} \frac{\|y-z\|_{W_{*}}}{\|y+z\|_{W_{*}}},
$$

and therefore if $W_{*}=W^{\perp}$ for some $W \subset X$, then $A\left(\left(W_{*}, Y\right),\left(W_{*}, Z\right)\right) \geq$ $a(Y / W, Z / W)$. In particular $Y / W$ and $Z / W$ do not form a direct sum in $X / W$ when $A\left(\left(W^{\perp}, Y\right),\left(W^{\perp}, Z\right)\right)=0$. If this is true for all $W, Y, Z$ with $W$ an infinite-codimensional subspace of $Y$ and of $Z$ then we deduce that $X$ is QHI.

By our previous description of special sequences in Gowers-Maurey's space, $X_{\mathrm{GM}}$ is an example of a reflexive space for which $A\left(\left(Y_{*}, Y\right),\left(Z_{*}, Z\right)\right)$ $=0$ for all infinite-dimensional QS-pairs $\left(Y_{*}, Y\right)$ and $\left(Z_{*}, Z\right)$ of $X$. Indeed, if $y, z, y^{*},-z^{*}$ are the odd and even parts respectively of adequate length $k$ special sequences, we have

$$
\|y-z\|\left\|y^{*}-z^{*}\right\| \simeq k / \sqrt{\log (k)},
$$

while

$$
\left|y^{*}(y)-z^{*}(z)\right| \simeq k .
$$

By construction, we may pick the terms of the special sequences close enough 
to $Y, Z, Y_{*}, Z_{*}$ respectively. It is not difficult to check that we may then perturb the almost biorthonormal system of special sequences so as to have $y \in Y, z \in Z, y^{*} \in Y_{*}, z^{*} \in Z_{*}$, and $y^{*}(z)=z^{*}(y)=0$, and preserving the estimates on $\|y-z\|\left\|y^{*}-z^{*}\right\|$ and $\left|y^{*}(y)-z^{*}(z)\right|$.

When $X$ is reflexive, the roles of $X$ and $X^{*}$ are interchangeable in the expression of $A$. Note that under reflexivity, the QHI property ([6, Corollary 4]) and the property of having an unconditional basis are self-dual properties.

1.3. FDD-block subspaces and FDD-block quotients of subspaces. We shall prove that a dichotomy theorem holds for quotients of subspaces which have a finite-dimensional decomposition (or FDD) relative to a given Schauder basis (or even a FDD) of a given Banach space; they seem to be the natural equivalent of block-subspaces considered in Gowers' dichotomy.

An interval of integers is the intersection of $\mathbb{N}$ with a bounded interval of $\mathbb{R}$. Two non-empty intervals $E_{1}$ and $E_{2}$ are said to be successive, written $E_{1}<E_{2}$, when $\max \left(E_{1}\right)<\min \left(E_{2}\right)$. A successive partition is a sequence $\left(E_{n}\right)_{n \in \mathbb{N}}$ of successive intervals forming a partition of $\mathbb{N}$.

Let $X$ be a Banach space with a finite-dimensional decomposition denoted $\left(B_{n}\right)_{n \in \mathbb{N}}$. When $x=\sum_{n \in \mathbb{N}} b_{n} \in X$ with $b_{n} \in B_{n}$ for all $n \in \mathbb{N}$, the support of $x$ is the set $\left\{i \in \mathbb{N}: b_{i} \neq 0\right\}$. The range of a vector is the smallest interval containing its support. The support of a subspace $Y$ of $X$ is the smallest set containing the supports of all vectors of $Y$. The range of $Y$, denoted $\operatorname{ran}(Y)$, is the smallest interval containing the support of $Y$. Two finitely supported subspaces $F$ and $G$ of $X$ with non-empty supports are successive when $\operatorname{ran}(F)<\operatorname{ran}(G)$.

An FDD-block subspace of $X$ is an infinite sum $\sum_{n \in \mathbb{N}} F_{n}$ of finitely supported (possibly zero-dimensional) subspaces $F_{n}$ of $X$ such that $\operatorname{ran}\left(F_{n}\right)$ $\subset E_{n}$ for all $n \in \mathbb{N}$, where $\left(E_{n}\right)_{n \in \mathbb{N}}$ is a successive partition. Therefore an FDD-block subspace is finite-dimensional or equipped with the FDD $\left(F_{n}\right)_{n \in I}$, where $I=\left\{n: F_{n} \neq\{0\}\right\}$.

An FDD-block quotient of $X$ is the quotient of $X$ by some FDD-block subspace $Y=\sum_{n \in \mathbb{N}} G_{n}$. An FDD-block quotient is finite-dimensional or equipped with the FDD $\left(C_{n}\right)_{n \in I}$ corresponding to the successive partition $\left(E_{n}\right)_{n \in \mathbb{N}}$ associated to $Y$, that is, $C_{n}=\left(\left[B_{i}: i \in E_{n}\right]+Y\right) / Y$ for all $n$, and $I=\left\{n: C_{n} \neq\{0\}\right\}$. Note that the space $X$ is an FDD-block quotient of itself.

An FDD-block quotient of a subspace of $X$ is a quotient of a subspace of $X$ of the form $\sum_{n \in \mathbb{N}} F_{n} / \sum_{n \in \mathbb{N}} G_{n}$, where $G_{n} \subset F_{n} \subset\left[B_{i}: i \in E_{n}\right]$ for all $n$, where $\left(E_{n}\right)_{n \in \mathbb{N}}$ is a successive partition. The space $\sum_{n \in \mathbb{N}} F_{n} / \sum_{n \in \mathbb{N}} G_{n}$ is naturally seen as an FDD-block subspace of $X / \sum_{n \in \mathbb{N}} G_{n}$, when $X / \sum_{n \in \mathbb{N}} G_{n}$ is equipped with the FDD corresponding to $\left(E_{n}\right)_{n \in \mathbb{N}}$. 
It is therefore clear that any FDD-block subspace (resp. quotient of a subspace) of an FDD-block subspace (resp. quotient of a subspace) of $X$ is again an FDD-block subspace (resp. quotient of a subspace) of $X$. Note also that by classical results, any subspace of $X$ contains, for any $\varepsilon>0$, a $1+\varepsilon$-isomorphic copy of a block subspace, and therefore of an FDD-block subspace (however, the similar result concerning QS-spaces does not seem to be clear). Considering FDD-block quotients of subspaces to study the structure of the class of QS-spaces is a natural counterpart of considering block-subspaces to study the structure of the class of subspaces.

Proposition 4. Let $X$ be a Banach space with a finite-dimensional decomposition. The following statements are equivalent:

(i) no FDD-block quotient of a subspace of $X$ is decomposable,

(ii) for any infinite-codimensional FDD-block subspace $W$ of $X$, the quotient $X / W$ is hereditarily indecomposable,

(iii) whenever $Y=\sum_{n \in \mathbb{N}} F_{n} / \sum_{n \in \mathbb{N}} G_{n}$ and $Y^{\prime}=\sum_{n \in \mathbb{N}} F_{n}^{\prime} / \sum_{n \in \mathbb{N}} G_{n}$ are infinite-dimensional FDD-block quotients of subspaces of $X$ with the same successive partition, the sum $Y+Y^{\prime}$ is not direct in $X / \sum_{n \in \mathbb{N}} G_{n}$.

When $X$ satisfies (i)-(iii) we shall say that $X$ is quotient hereditarily indecomposable restricted to FDD-block subspaces, or for short, has the restricted QHI property.

Proof. (ii) $\Rightarrow$ (i) is immediate. If (iii) is false then the FDD-block quotient of a subspace $Y+Y^{\prime}=\sum_{n \in \mathbb{N}}\left(F_{n}+F_{n}^{\prime}\right) / \sum_{n \in \mathbb{N}} G_{n}$ is decomposable, contradicting (i). Finally, assume (ii) is false, i.e. $Z / W \oplus Z^{\prime} / W$ forms a direct sum of infinite-dimensional subspaces in $X / W$ for some infinite-codimensional FDD-block subspace $W=\sum_{n \in \mathbb{N}} G_{n}$ and some subspaces $Z$ and $Z^{\prime}$, and let $\left(E_{n}\right)_{n \in \mathbb{N}}$ be a successive partition associated to $W$. We may find sequences $\left(z_{n}\right)_{n \in \mathbb{N}}$ and $\left(z_{n}^{\prime}\right)_{n \in \mathbb{N}}$, and a partition $\left(N_{n}\right)_{n \in \mathbb{N}}$ of $\mathbb{N}$ into successive intervals, such that for all $n \in \mathbb{N}, \operatorname{ran}\left(z_{n}, z_{n}^{\prime}\right) \subset \bigcup_{i \in N_{n}} E_{i}$, and $d\left(z_{n}, Z\right)$ and $d\left(z_{n}^{\prime}, Z^{\prime}\right)$ converge to 0 sufficiently fast so that $\left(\left[z_{n}\right]_{n \in \mathbb{N}}+W\right) / W \oplus\left(\left[z_{n}^{\prime}\right]_{n \in \mathbb{N}}+W\right) / W$ is still direct in $X / W$. Define $H_{n}=\sum_{i \in N_{n}} G_{i}$ for all $n \in \mathbb{N}$; then $\left(\sum_{n \in \mathbb{N}}\left(H_{n}+\left[z_{n}\right]\right)\right) / \sum_{n \in \mathbb{N}} H_{n}$ and $\left(\sum_{n \in \mathbb{N}}\left(H_{n}+\left[z_{n}^{\prime}\right]\right)\right) / \sum_{n \in \mathbb{N}} H_{n}$ form a direct sum, with successive partition $\left(\bigcup_{i \in N_{n}} E_{i}\right)_{n \in \mathbb{N}}$, contradicting (iii).

FDD-block quotients of subspaces still capture enough information about the structure of the space: a space which has the restricted QHI property is in particular hereditarily indecomposable by (ii), and by (i), any of its infinitedimensional FDD-block quotients of subspaces has again the restricted QHI property. The next proposition also shows that the restricted QHI property has similar self-dual properties to the QHI property. 
Proposition 5. Let $X$ be a Banach space with a shrinking finite-dimensional decomposition such that $X^{*}$ has the restricted QHI property. Then $X$ has the restricted QHI property.

Proof. Let $Y=\sum_{n \in \mathbb{N}} F_{n} / \sum_{n \in \mathbb{N}} G_{n}$ be an infinite-dimensional FDDblock quotient of a subspace of $X$, with successive partition $\left(E_{n}\right)_{n \in \mathbb{N}}$. For each $n \in \mathbb{N}$, let $X_{n}^{*}$ be the space of vectors in $X^{*}$ with range included in $E_{n}$. Then

$$
Y^{*}=\left(\sum_{n \in \mathbb{N}} G_{n}\right)^{\perp} /\left(\sum_{n \in \mathbb{N}} F_{n}\right)^{\perp}=\left(\sum_{n \in \mathbb{N}}\left(G_{n}^{\perp} \cap X_{n}^{*}\right)\right) /\left(\sum_{n \in \mathbb{N}}\left(F_{n}^{\perp} \cap X_{n}^{*}\right)\right),
$$

since $\left(E_{n}\right)_{n \in \mathbb{N}}$ is a partition of $\mathbb{N}$. So $Y^{*}$ is an FDD-block quotient of a subspace of $X^{*}$. Therefore according to the first characterization in Proposition 4 , if $X$ does not have the restricted QHI property, then $X^{*}$ does not have the restricted QHI property.

In consequence, we note that if $X$ is a reflexive Banach space with the restricted QHI property, then $X$ has HI dual and is saturated with subspaces with HI dual. Indeed, every FDD-block subspace of $X$ has HI dual.

We are now in a position to state the main result of this paper.

THEOREM 6. Every infinite-dimensional Banach space has a quotient of a subspace, $Y$, with one of the following two properties, which are mutually exclusive and both possible:

(i) $Y$ has an unconditional basis,

(ii) $Y$ has the restricted QHI property.

We give a few comments on the reasons we needed to impose a restriction on the QHI property. Our proof is based on a method of "combinatorial forcing" (see Todorcevic's course [3]). It will enable us to prove a general dichotomy result for closed properties of FDD-block quotients of subspaces, seen as sequences of finite-dimensional successive QS-blocks (this will be defined precisely in the next section), with the product of the discrete topology on the set of QS-blocks. This applies more or less directly to yield Theorem 6 .

As we see them, these methods rely on defining infinite sequences of elements which may be correctly approximated by finite sequences; a notion of succession is needed, i.e. finite sequences are extended to infinite sequences in a way that does not "affect" the properties implied by the finite part.

Our proof was inspired by a simplification by B. Maurey of this method in the case of block-subspaces of a space with a Schauder basis, where a less restrictive setting may be used, based on replacing $X$ by a countable dense subset [12].

It did not seem possible to repeat Maurey's proof exactly to study QS-spaces. Therefore we needed to restrict our study to particular QSspaces which may be canonically associated to infinite sequences of "finite- 
dimensional blocks" which are successive in some sense. For technical reasons, the countable dense subset must be replaced by a net whose intersection with the set of predecessors of a given block is always finite. Up to perturbations, the restriction to a net is not essential, but the need for some notion of succession seems to be, and this justifies why we could not obtain "quotient hereditarily indecomposable" in the second part of the conclusion of Theorem 6. Actually some examples indicate that FDD-block quotients of subspaces may behave differently from general quotients of subspaces. We refer to the final section for this fact.

2. Proof of the theorem. To prove Theorem 6 , we may consider a Banach space $X$ with a Schauder basis $\left(e_{n}\right)$. We denote by $\left(e_{n}^{*}\right)$ its dual basis, and by $X_{*}$ the closed linear span of $\left(e_{n}^{*}\right)_{n \in \mathbb{N}}$. We may also assume that the basis is bimonotone. We shall consider supports and ranges of vectors or subspaces of $X$ and of $X_{*}$ with respect to these canonical bases.

We choose to represent blocks forming quotients of subspaces of $X$ as pairs formed by a finite-dimensional subspace $F$ of $X$ and a finitedimensional subspace $F_{*}$ of $X_{*}$, with $F_{*}^{\perp} \cap\left[e_{n}: n \in \operatorname{ran}\left(F, F_{*}\right)\right] \subset F$. Pairs $(F, G)$ of finite-dimensional subspaces of $X$ with $G \subset F$ would also have been a possible representation. Our choice will save us from some technicalities (successive pairs in our setting are pairs whose supports are necessarily immediately successive). It will also preserve, in our proofs, the symmetry between the roles played by $X$ and $X^{*}$ in the reflexive case. This symmetry is apparent in our main result, and we felt it worth emphasizing in our demonstration.

2.1. Blockings of QS-pairs. If $Y \subset X$ and $Y_{*} \subset X_{*}$, the range of $\left(Y_{*}, Y\right)$ is the smallest interval containing the ranges of $Y$ and of $Y_{*}$. The set of finitely supported subspaces of $X$ is denoted $F(X)$, and that of finitely supported subspaces of $X_{*}$ is denoted $F\left(X_{*}\right)$. A $Q S$-block (or block) is a pair $\left(F_{*}, F\right) \in F\left(X_{*}\right) \times F(X)$ such that $F_{*}^{\perp} \cap\left[e_{n}: n \in E\right] \subset F$, where $E:=\operatorname{ran}\left(F_{*}, F\right)$. The set of blocks is denoted $\mathcal{F}(X)$. The dimension of $\left(F_{*}, F\right)$ is the dimension of $F /\left(F_{*}^{\perp} \cap\left[e_{n}: n \in E\right]\right)$. Two blocks $\left(F_{*}, F\right)$ and $\left(G_{*}, G\right)$ are said to be successive if $\min \left(\operatorname{ran}\left(G_{*}, G\right)\right)=\max \left(\operatorname{ran}\left(F_{*}, F\right)\right)+1$, and we write $\left(F_{*}, F\right)<\left(G_{*}, G\right)$ (note the technical difference from the usual notion of succession).

We note that when $\mathcal{Y}=\left(Y_{n *}, Y_{n}\right)_{n \in \mathbb{N}}$ is a sequence of successive blocks whose ranges partition $\mathbb{N}$ (equivalently, such that $\min \left(\operatorname{ran}\left(Y_{1 *}, Y_{1}\right)\right)=1$ ), the spaces $Y=\sum_{n \in \mathbb{N}} Y_{n}$ and $Y_{*}=\sum_{n \in \mathbb{N}} Y_{n *}$ satisfy $Y_{*}^{\perp} \subset Y$. We shall then say that $\left(Y_{*}, Y\right)$ is the QS-pair associated to $\mathcal{Y}$, and that $\mathcal{Y}$ is infinitedimensional whenever the QS-space $Y / Y_{*}^{\perp}$ is infinite-dimensional. Note that the space $Y / Y_{*}^{\perp}$ is an FDD-block quotient of a subspace of $X$. 
If $\left(F_{*}, F\right)$ is a block and $\left(Y_{n *}, Y_{n}\right)_{n \in I}$ is a finite or an infinite sequence of successive blocks, and if there exists an interval $E \subset I$ such that $F \subset$ $\sum_{n \in E} Y_{n}$ and $F_{*} \subset \sum_{n \in E} Y_{n *}$, then we shall say that $\left(F_{*}, F\right)$ is a block of $\left(Y_{n *}, Y_{n}\right)_{n \in I}$.

We now define a relation of "blocking" between sequences of successive blocks.

Definition 7. Let $\left(Y_{n *}, Y_{n}\right)_{n}$ and $\left(Z_{i *}, Z_{i}\right)_{i}$ be finite or infinite sequences of successive blocks. If for any $i,\left(Z_{i *}, Z_{i}\right)$ is a block of $\left(Y_{n *}, Y_{n}\right)_{n}$, then we shall say that $\left(Z_{i *}, Z_{i}\right)_{i}$ is a blocking of $\left(Y_{n *}, Y_{n}\right)_{n}$.

If $\mathcal{Z}=\left(Z_{i *}, Z_{i}\right)_{i \in \mathbb{N}}$ and $\mathcal{Y}=\left(Y_{n *}, Y_{n}\right)_{n \in \mathbb{N}}$ are infinite sequences of successive blocks whose ranges partition $\mathbb{N}$, then we shall write $\mathcal{Z} \leq \mathcal{Y}$ if $\mathcal{Z}$ is a blocking of $\mathcal{Y}$. This means that there exists a partition $\left\{N_{i}: i \in \mathbb{N}\right\}$ of $\mathbb{N}$ into successive intervals such that, for all $i \in \mathbb{N}, \operatorname{ran}\left(Z_{i *}, Z_{i}\right)=$ $\bigcup_{n \in N_{i}} \operatorname{ran}\left(Y_{n *}, Y_{n}\right)$ and $\left(Z_{i *}, Z_{i}\right)$ is a block of $\left(Y_{n *}, Y_{n}\right)_{n \in N_{i}}$.

We note that $\leq$ is an order relation. Clearly, when $\mathcal{Z} \leq \mathcal{Y}$, the associated QS-pairs $\left(Z_{*}, Z\right)$ and $\left(Y_{*}, Y\right)$ satisfy $\left(Z_{*}, Z\right) \subset\left(Y_{*}, Y\right)$.

For any two sequences $\mathcal{Y}$ and $\mathcal{Z}$, we define

$$
A(\mathcal{Y}, \mathcal{Z})=A\left(\left(Y_{*}, Y\right),\left(Z_{*}, Z\right)\right)
$$

where $\left(Y_{*}, Y\right)$ and $\left(Z_{*}, Z\right)$ are the associated QS-pairs.

Lemma 8. Let $\mathcal{Y}=\left(Y_{n *}, Y_{n}\right)_{n \in \mathbb{N}}$ be an infinite-dimensional, successive sequence of blocks whose ranges partition $\mathbb{N}$. Let $\left(Y_{*}, Y\right)$ be the associated $Q S$ pair. Assume that $A(\mathcal{U}, \mathcal{V})=0$ whenever $\mathcal{U}, \mathcal{V} \leq \mathcal{Y}$ are infinite-dimensional, successive sequences of blocks whose ranges are equal and partition $\mathbb{N}$. Then $Y / Y_{*}^{\perp}$ has the restricted QHI property.

Proof. The proof is based on the natural identification between sequences of blocks of $Y / Y_{*}^{\perp}$ with its natural finite-dimensional decomposition, and sequences of blocks of $X$ which are blockings of $\left(Y_{*}, Y\right)$. Indeed, consider two infinite-dimensional FDD-block quotients of subspaces of $Y / Y_{*}^{\perp}$ which are of the form $Z=\sum_{n \in \mathbb{N}} F_{n} / \sum_{n \in \mathbb{N}} G_{n}$ and $Z^{\prime}=\sum_{n \in \mathbb{N}} F_{n}^{\prime} / \sum_{n \in \mathbb{N}} G_{n}$, with successive partition $\left(E_{n}\right)_{n \in \mathbb{N}}$. By definition for all $n \in \mathbb{N}, G_{n} \subset F_{n} \subset$ $\left(\sum_{k \in E_{n}} Y_{k}+Y_{*}^{\perp}\right) / Y_{*}^{\perp}$, and let $I_{n}=\operatorname{ran}\left(\sum_{k \in E_{n}} Y_{k}\right)$. Therefore we may find $A_{n}, B_{n}$ such that

$$
\left(\sum_{k \in E_{n}} Y_{k *}\right)^{\perp} \cap\left[e_{i}: i \in I_{n}\right] \subset B_{n} \subset A_{n} \subset \sum_{k \in E_{n}} Y_{n},
$$

and such that $G_{n}=\left(B_{n}+Y_{*}^{\perp}\right) / Y_{*}^{\perp}$ and $F_{n}=\left(A_{n}+Y_{*}^{\perp}\right) / Y_{*}^{\perp}$. We define some subspaces $A_{n}^{\prime}$ associated to the spaces $F_{n}^{\prime}$ in a similar way.

We therefore have the identification

$$
Z \simeq \overline{\sum_{n \in \mathbb{N}} A_{n}+Y_{*}^{\perp}} / \overline{\sum_{n \in \mathbb{N}} B_{n}+Y_{*}^{\perp}}=\sum_{n \in \mathbb{N}} A_{n} / \sum_{n \in \mathbb{N}} B_{n},
$$


which is by construction an FDD-block quotient of a subspace of $X$ corresponding to a blocking of $\mathcal{Y}$. Indeed, let $B_{n *}=B_{n}^{\perp} \cap\left[e_{i}: i \in I_{n}\right]$, and let $\mathcal{Z}=$ $\left(B_{n *}, A_{n}\right)_{n \in \mathbb{N}}$. Then the associated QS-space is $\sum_{n \in \mathbb{N}} A_{n} /\left(\sum_{n \in \mathbb{N}} B_{n *}\right)^{\perp}=$ $\sum_{n \in \mathbb{N}} A_{n} / \sum_{n \in \mathbb{N}} B_{n}$.

We have the similar identification for $Z^{\prime}$ and let $\mathcal{Z}^{\prime}=\left(B_{n *}, A_{n}^{\prime}\right)_{n \in \mathbb{N}}$. Since $\mathcal{Z} \leq \mathcal{Y}$ and $\mathcal{Z}^{\prime} \leq \mathcal{Y}$, it follows that $A\left(\mathcal{Z}, \mathcal{Z}^{\prime}\right)=0$. This means that the spaces $\sum_{n \in \mathbb{N}} A_{n} / \sum_{n \in \mathbb{N}} B_{n}$ and $\sum_{n \in \mathbb{N}} A_{n}^{\prime} / \sum_{n \in \mathbb{N}} B_{n}$ do not form a direct sum in $Y / \sum_{n \in \mathbb{N}} B_{n}$, and therefore $Z$ and $Z^{\prime}$ do not form a direct sum in the space $\left(Y / Y_{*}^{\perp}\right) / \sum_{n \in \mathbb{N}} G_{n}$. Therefore (iii) of Proposition 4 is satisfied.

Before stating more definitions, we need to realize a reduction to a net $\mathcal{R}$ of blocks with some finiteness property which will be crucial for our combinatorial method.

For $F, G$ in $F(X)$, we let $d_{\mathrm{H}}(F, G)$ be the Hausdorff distance between the unit spheres $S_{F}$ of $F$ and $S_{G}$ of $G, d_{H}(F, G)=\max _{x \in S_{F}} d\left(x, S_{G}\right) \vee$ $\max _{y \in S_{G}} d\left(y, S_{F}\right)$. Modifying a definition from [7], we define a distance $d$ on $F(X)$ by

$$
d(F, G)=\min \left(1,2 k \sqrt{k} d_{\mathrm{H}}(F, G)\right)
$$

if $\operatorname{dim} F=\operatorname{dim} G=k$ and $\operatorname{ran}(F)=\operatorname{ran}(G)$, and $d(F, G)=1$ otherwise.

We finally define a distance $\delta$ on $\mathcal{F}(X)$ by

$$
\delta\left(\left(F_{*}, F\right),\left(G_{*}, G\right)\right)=\max \left(d(F, G), d\left(F_{*}^{\perp} \cap X_{0}, G_{*}^{\perp} \cap X_{0}\right)\right)
$$

when $\operatorname{ran}\left(F_{*}, F\right)=\operatorname{ran}\left(G_{*}, G\right)$ and $X_{0}=\left[e_{i}: i \in \operatorname{ran}\left(F_{*}, F\right)\right]$, and we let $\delta\left(\left(F_{*}, F\right),\left(G_{*}, G\right)\right)=1$ otherwise.

The critical result concerning this distance is contained in the next lemma.

Lemma 9. Let $0<\varepsilon<1$ and let $\left(\delta_{n}\right)_{n}$ be a positive sequence such that $\sum_{n \in \mathbb{N}} \delta_{n} \leq \varepsilon$. Let $\left(F_{n *}, F_{n}\right)_{n \in \mathbb{N}}$ and $\left(G_{n *}, G_{n}\right)_{n \in \mathbb{N}}$ be successive sequences of blocks such that for all $n \in \mathbb{N}, \delta\left(\left(F_{n *}, F_{n}\right),\left(G_{n *}, G_{n}\right)\right) \leq \delta_{n}$, and, for $n \in \mathbb{N}$, let $X_{n}$ be the space $\left[e_{i}: i \in \operatorname{ran}\left(F_{n *}, F_{n}\right)\right]$. Then there exists a map $T$ : $\sum_{n \in \mathbb{N}} F_{n} \rightarrow \sum_{n \in \mathbb{N}} G_{n}$ such that $T\left(F_{n}\right)=G_{n}$ and $T\left(F_{n *}^{\perp} \cap X_{n}\right)=G_{n *}^{\perp} \cap X_{n}$ for all $n \in \mathbb{N}$, and $\|T x-x\| \leq \varepsilon\|x\|$ for any $x \in \sum_{n \in \mathbb{N}} F_{n}$.

Proof. Let $k=\operatorname{dim} F_{1}=\operatorname{dim} G_{1}$ and let $l=\operatorname{dim} F_{1 *}^{\perp} \cap X_{1}=\operatorname{dim} G_{1 *}^{\perp} \cap X_{1}$. By classical results, the Banach-Mazur distance of $F_{1}$ to $l_{2}^{k}$ is at most $\sqrt{k}$, so we may pick a normalized basis $f_{1}, \ldots, f_{k}$ of $F_{1}$ with basis constant at most $\sqrt{k}$ and such that $f_{1}, \ldots, f_{l}$ is a basis of $F_{1 *}^{\perp} \cap X_{1}$. By the expression of $\delta$, we have $d_{\mathrm{H}}\left(F_{1 *}^{\perp} \cap X_{1}, G_{1 *}^{\perp} \cap X_{1}\right) \leq \delta_{1} / 2 k \sqrt{k}$, therefore for $1 \leq i \leq l$, there exists some $g_{i} \in G_{1 *}^{\perp} \cap X_{1}$ with $\left\|g_{i}-f_{i}\right\| \leq \delta_{1} / 2 k \sqrt{k}$. Likewise we find for $l<i \leq k$ some $g_{i} \in G_{1}$ with the same condition on $\left\|g_{i}-f_{i}\right\|$.

By [10, Prop. 1.a.9], $\left(g_{i}\right)_{1 \leq i \leq k}$ is a basis of $G_{1}$, and furthermore, if $T_{1}$ : $F_{1} \rightarrow G_{1}$ is defined by $T_{1}\left(f_{i}\right)=g_{i}$ for all $1 \leq i \leq k$, we have, for any $x \in F_{1}$, 
$x=\sum_{i=1}^{k} a_{i} f_{i}$,

$$
\left\|T_{1} x-x\right\| \leq \sum_{i=1}^{k}\left|a_{i}\right|\left\|f_{i}-g_{i}\right\| \leq 2 \sqrt{k}\|x\| k\left(\delta_{1} / 2 k \sqrt{k}\right)=\delta_{1}\|x\| .
$$

Repeating this construction on each $F_{n}$, let $T_{n}$ be the associated map from $F_{n}$ onto $G_{n}$ with $T_{n}\left(F_{n *}^{\perp} \cap X_{n}\right)=G_{n *}^{\perp} \cap X_{n}$, and let $T$ be defined on $\sum_{n \in \mathbb{N}} F_{n}$ by $T_{\mid F_{n}}=T_{n}$ for all $n \in \mathbb{N}$. For any $x=\sum_{n \in \mathbb{N}} x_{n}$ with $x_{n} \in F_{n}$, we have

$$
\|T x-x\| \leq \sum_{n \in \mathbb{N}}\left\|T_{n} x_{n}-x_{n}\right\| \leq \sum_{n \in \mathbb{N}} \delta_{n}\left\|x_{n}\right\| \leq \varepsilon\|x\|,
$$

by bimonotonicity of the basis.

For $N \in \mathbb{N}$, we let $\mathcal{F}_{N}(X)$ be the set of elements $\left(F_{*}, F\right)$ of $\mathcal{F}(X)$ such that $\max \left(\operatorname{ran}\left(F_{*}, F\right)\right)=N$. Fixing a decreasing positive sequence $\left(\delta_{n}\right)_{n \in \mathbb{N}}$ such that $\delta_{n} \leq 2^{-n}$ for every $n \in \mathbb{N}$, we choose a subset $\mathcal{R} \subset \mathcal{F}(X)$ with the following properties:

(i) $\mathcal{R} \cap \mathcal{F}_{N}(X)$ is a finite $\delta_{N}$-net for $\mathcal{F}_{N}(X)$,

(ii) whenever $\left(F_{1 *}, F_{1}\right)<\cdots<\left(F_{k *}, F_{k}\right)$ belong to $\mathcal{R}$, it follows that $\left(F_{1 *}+\cdots+F_{k *}, F_{1}+\cdots+F_{k}\right)$ belongs to $\mathcal{R}$.

(iii) for any $\left(F_{*}, F\right) \in \mathcal{R} \cap \mathcal{F}_{N}(X), \mathcal{R} \cap \mathcal{F}_{F_{*}, F}$ is a $\delta_{N}$-net for $\mathcal{F}_{F_{*}, F}$, where $\mathcal{F}_{F_{*}, F}:=\left\{\left(G_{*}, G\right) \in \mathcal{F}_{N}(X):(G \subset F) \wedge\left(G_{*} \subset F_{*}\right)\right\}$.

(iv) for any $\left(F_{*}, F\right) \in \mathcal{R} \cap \mathcal{F}_{N}(X), \mathcal{R} \cap \mathcal{F}_{F}^{F_{*}}$ is a $\delta_{N}$-net for $\mathcal{F}_{F}^{F_{*}}$, where $\mathcal{F}_{F}^{F_{*}}:=\left\{\left(G_{*}, F\right) \in \mathcal{F}_{N}(X): G_{*} \subset F_{*}\right\}$,

(v) for any $\left(F_{*}, F\right) \in \mathcal{R} \cap \mathcal{F}_{N}(X), \mathcal{R} \cap \mathcal{F}_{F_{*}}^{F}$ is a $\delta_{N}$-net for $\mathcal{F}_{F_{*}}^{F}$, where $\mathcal{F}_{F_{*}}^{F}:=\left\{\left(F_{*}, G\right) \in \mathcal{F}_{N}(X): G \subset F\right\}$,

(vi) if $\left(F_{*}, F\right) \in \mathcal{R}$ then $\left(F^{\perp} \cap\left[e_{i}^{*}: i \in E\right], F_{*}^{\perp} \cap\left[e_{i}: i \in E\right]\right) \in \mathcal{R}$, where $E=\operatorname{ran}\left(F_{*}, F\right)$.

An $\mathcal{R}$-block will denote a block in $\mathcal{R}$. In the following, blocks will always be $\mathcal{R}$-blocks, unless specified otherwise.

We denote by $\mathrm{QS}^{<\omega}(X)$ (resp. $\mathrm{QS}_{0}^{<\omega}(X)$ ) the set of finite sequences of successive $\mathcal{R}$-blocks $\left(F_{n *}, F_{n}\right)_{n}$ (resp. for which $\left.\min \left(\operatorname{ran}\left(F_{1 *}, F_{1}\right)\right)=1\right)$.

The set $\mathrm{QS}^{\omega}(X)$ (resp. $\left.\mathrm{QS}_{0}^{\omega}(X)\right)$ is the space of infinite sequences of successive $\mathcal{R}$-blocks $\mathcal{Y}=\left(Y_{n *}, Y_{n}\right)_{n}$ (resp. for which $\left.\min \left(\operatorname{ran}\left(Y_{1 *}, Y_{1}\right)\right)=1\right)$. If $\left(Y_{n *}, Y_{n}\right)_{n}$ is an element of $\mathrm{QS}_{0}^{\omega}(X)$, the partition of $\left(Y_{n *}, Y_{n}\right)_{n}$ is the sequence $\left(\operatorname{ran}\left(Y_{n *}, Y_{n}\right)\right)_{n \in \mathbb{N}}$, which is a partition of $\mathbb{N}$. The space $\sum_{n \in \mathbb{N}} Y_{n}$ will be denoted $Y$, and $Y_{*}$ will denote $\sum_{n \in \mathbb{N}} Y_{n *}$. As already observed, the relation $Y_{*}^{\perp} \subset Y$ ensures that $Y / Y_{*}^{\perp}$ is an FDD-block quotient of a subspace of $X$. We let $\mathrm{QS}(X) \subset \mathrm{QS}_{0}^{\omega}(X)$ be the set of sequences which are infinite-dimensional, that is, such that the QS-space $Y / Y_{*}^{\perp}$ is infinite-dimensional. 
If $E$ is an interval of integers, and $\left(Y_{n *}, Y_{n}\right)_{n \in I}$ is a finite or an infinite sequence of successive $\mathcal{R}$-blocks, we shall say that $\left(Y_{n *}, Y_{n}\right)_{n \in I}$ is well-placed with respect to $E$ if there exists $m \in I$ such that $\min \left(\operatorname{ran}\left(Y_{m *}, Y_{m}\right)\right)=$ $\max E+1$. The set of sequences of $\mathrm{QS}(X)$ which are well-placed with respect to $E$ is denoted $\mathrm{QS}_{E}(X)$.

We now define a relation of "tail blocking" on $\mathrm{QS}(X)$.

Definition 10. Let $\mathcal{Z}, \mathcal{Y} \in \mathrm{QS}(X)$. If $E$ is an interval of $\mathbb{N}$, and $\left(Z_{i *}, Z_{i}\right)_{i \geq p}$ is a blocking of $\left(Y_{n *}, Y_{n}\right)_{n \geq m}$ with $\min \left(\operatorname{ran}\left(Z_{p *}, Z_{p}\right)\right)=\min \left(\operatorname{ran}\left(Y_{m *}, Y_{m}\right)\right)=$ $\max E+1$, then we shall write $\mathcal{Z} \leq^{E} \mathcal{Y}$.

Note that if $\mathcal{Z} \leq^{E} \mathcal{Y}$ then necessarily $\mathcal{Z}$ and $\mathcal{Y}$ are well-placed with respect to $E$. It is also clear that $\leq^{E}$ is a preorder relation, and $\mathcal{W} \leq{ }^{E} \mathcal{Y}$ whenever $\mathcal{W}$ and $\mathcal{Y}$ are well-placed with respect to $E$ and $\mathcal{W} \leq \mathcal{Y}$.

We shall need the following easy lemma.

Lemma 11. Let $E$ be an interval of $\mathbb{N}$, and $\mathcal{Y}, \mathcal{Z} \in \mathrm{QS}_{E}(X)$. Assume $\mathcal{Z} \leq^{E} \mathcal{Y}$. Then there exists $\mathcal{W} \in \mathrm{QS}_{E}(X)$ such that $\mathcal{W} \leq \mathcal{Y}$ and $\mathcal{W} \leq{ }^{E} \mathcal{Z}$.

Proof. Let $\min \left(\operatorname{ran}\left(Y_{m *}, Y_{m}\right)\right)=\max E+1=\min \left(\operatorname{ran}\left(Z_{p *}, Z_{p}\right)\right)$ for some $m, p$. We define $\left(W_{n *}, W_{n}\right)=\left(Y_{n *}, Y_{n}\right)$ if $n<m$ and $\left(W_{n *}, W_{n}\right)=$ $\left(Z_{(n-m+p) *}, Z_{n-m+p}\right)$ if $n \geq m$.

Definition 12. Let $P \subset \mathrm{QS}_{E}(X)$. We say that $P$ is $\leq^{E}$-hereditary if whenever $\mathcal{Y} \in P$ and $\mathcal{Z} \leq^{E} \mathcal{Y}$, then $\mathcal{Z} \in P$. We say that $P$ is $\leq^{E}$-large if it is $\leq^{E}$-hereditary and whenever $\mathcal{Y} \in \mathrm{QS}_{E}(X)$, there exists $\mathcal{Z} \leq \mathcal{Y}$ such that $\mathcal{Z} \in P$.

2.2. A game for QS-pairs. Our proof will be based on an "oriented QSpairs" Gowers game $G_{\mathcal{A}}^{\mathcal{Y}}$ associated to some subset $\mathcal{A}$ of $\mathrm{QS}(X) \times\{-1,1\}^{\omega}$ and to some $\mathcal{Y} \in \operatorname{QS}(X)$, and defined as follows. Player 1 plays some $\mathcal{W}_{1} \leq \mathcal{Y}$. Player 2 plays some sign $\varepsilon_{1} \in\{-1,1\}$ and some block $\left(U_{1 *}, U_{1}\right)$ which is a block of $\mathcal{W}_{1}$ with $\min \left(\operatorname{ran}\left(U_{1 *}, U_{1}\right)\right)=1$.

At step $n$, Player 1 plays some $\mathcal{W}_{n} \leq \mathcal{Y}$ which is well-placed with respect to $\operatorname{ran}\left(U_{(n-1) *}, U_{n-1}\right)$. Player 2 plays some sign $\varepsilon_{n} \in\{-1,1\}$ and some block $\left(U_{n *}, U_{n}\right)$ of $\mathcal{W}_{n}$ which is successive with respect to $\left(U_{(n-1) *}, U_{n-1}\right)$.

Player 2 wins the game if he produces an infinite sequence $\left(U_{n *}, U_{n}, \varepsilon_{n}\right)_{n}$ which is in $\mathcal{A}$.

In our application we shall use this game for the set $\mathcal{A}_{\delta}$ for some $\delta>0$, defined as the set of $\left(U_{n *}, U_{n}, \varepsilon_{n}\right)_{n}$ such that there exist $n \in \mathbb{N}$ and $u_{k} \in U_{k}$, $u_{k}^{*} \in U_{k *}, 1 \leq k \leq n$, such that

$$
\left\|\sum_{k=1}^{n} u_{k}\right\||| \sum_{k=1}^{n} u_{k}^{*} \|<\delta\left|\sum_{k=1}^{n} \varepsilon_{k-1} u_{k}^{*}\left(u_{k}\right)\right|,
$$

where $\varepsilon_{0}=1$ is fixed. 
A state $s$ will be an element of $\mathrm{QS}^{<\omega}(X) \times\{-1,1\}^{<\omega}$, where the two sequences are of equal length denoted $|s|$. The set of states will be denoted $S$. When $\mathcal{Y}$ is well-placed with respect to $\left(U_{i *}, U_{i}\right)_{i<k}$ and $\left(\varepsilon_{i}\right)_{i<k}$ is a sequence of signs, we define in an obvious way the game $G_{\mathcal{A}}^{\mathcal{Y}}(s)$, where $s$ is the state $\left(U_{n *}, U_{n}, \varepsilon_{n}\right)_{n<k}$ : just rename steps $1,2, \ldots$ in the new game as $k, k+1, \ldots$ and then apply the same definition as above; this is the game $G_{\mathcal{A}}^{\mathcal{Y}}$ starting from position $s$.

If $s=\left(U_{n *}, U_{n}, \varepsilon_{n}\right)_{n<k}$, then $\operatorname{ran}(s)$ will denote $\operatorname{ran}\left(\left(U_{n *}, U_{n}\right)_{n<k}\right)$, and to simplify the notation we also let $\mathrm{QS}_{s}(X)$ stand for $\mathrm{QS}_{\mathrm{ran}(s)}(X)$, and $\leq^{s}$ for $\leq^{\operatorname{ran}(s)}$; moreover, "successive to $s$ " will mean "successive to $\left(U_{(k-1) *}, U_{k-1}\right)$ ".

In the following, we fix some subset $\mathcal{A}$ of $\mathrm{QS}(X) \times\{-1,1\}^{\omega}$. Our next definition is the first step of the method of "combinatorial forcing" on $\operatorname{QS}(X)$.

Definition 13. Let $s$ be a state, and let $\mathcal{Y} \in \mathrm{QS}_{s}(X)$.

The state $s$ accepts $\mathcal{Y}$ if Player 2 has a winning strategy for the game $G_{\mathcal{A}}^{\mathcal{Y}}(s)$.

The state $s$ rejects $\mathcal{Y}$ if it accepts no $\mathcal{Z} \leq \mathcal{Y}$.

The state $s$ decides $\mathcal{Y}$ if it accepts or rejects $\mathcal{Y}$.

Lemma 14. Let $s$ be a state.

- The set of $\mathcal{Y}$ in $\mathrm{QS}_{s}(X)$ such that $s$ accepts $\mathcal{Y}$ (resp. rejects $\mathcal{Y}$ ) is $\leq^{s}$-hereditary.

- The set of $\mathcal{Y}$ in $\operatorname{QS}_{s}(X)$ such that $s$ decides $\mathcal{Y}$ is $\leq^{s}$-large.

Proof. Assume $s$ accepts $\mathcal{Y}$. Let $\mathcal{Z}$ be such that $\mathcal{Z} \leq^{s} \mathcal{Y}$. Let $\mathcal{W}=\mathcal{W}_{n} \leq$ $\mathcal{Z}$ be a move for Player 1 in $G_{\mathcal{A}}^{\mathcal{Z}}(s)$ at step $n$. By Lemma 11, we may find $\mathcal{V} \leq \mathcal{Y}$ with $\mathcal{V} \leq s \mathcal{W}$, in particular $\mathcal{V}$ is well-placed with respect to $\operatorname{ran}(s)$. Therefore $\mathcal{V}_{n}=\mathcal{V}$ is an admissible move for Player 1 in $G_{\mathcal{A}}^{\mathcal{Y}}(s)$. Since $s$ accepts $\mathcal{Y}$, a move $\left(U_{n *}, U_{n}, \varepsilon_{n}\right)$ for Player 2 is prescribed by the winning strategy for $G_{\mathcal{A}}^{\mathcal{Y}}(s)$. This move is admissible for Player 2 in $G_{\mathcal{A}}^{\mathcal{Z}}(s)$, since $\left(U_{n *}, U_{n}\right)$ is successive to $s$ and therefore is a block of $\mathcal{W}$. We have therefore described a winning strategy for Player 2 in the game $G_{\mathcal{A}}^{\mathcal{Z}}(s)$, which means that $s$ accepts $\mathcal{Z}$.

Assume now that $s$ rejects $\mathcal{Y} \in \mathrm{QS}_{s}(X)$ while it does not reject $\mathcal{Z} \leq^{s} \mathcal{Y}$. We may assume that $s$ accepts $\mathcal{Z}$. We get a contradiction by using Lemma 11 to find some element $\mathcal{W} \in \operatorname{QS}_{s}(X)$ such that $\mathcal{W} \leq \mathcal{Y}$ and $\mathcal{W} \leq^{s} \mathcal{Z}$.

It follows that the set of $\mathcal{Y}$ in $\operatorname{QS}_{s}(X)$ such that $s$ decides $\mathcal{Y}$ is $\leq^{s}$ hereditary. Finally, if $\mathcal{Y} \in \mathrm{QS}_{s}(X)$, then either $s$ rejects $\mathcal{Y}$, or $s$ accepts some $\mathcal{Z} \leq \mathcal{Y}$; this implies $\leq^{s}$-largeness.

Lemma 15 (Stabilization principle). For any $\mathcal{W} \in \mathrm{QS}(X)$, there exists $\mathcal{Y} \leq \mathcal{W}$ such that whenever $\left(Z_{n *}, Z_{n}\right)_{n \leq k} \in \mathrm{QS}_{0}^{<\omega}(X)$ is a blocking of $\mathcal{Y}$, and $\left(\varepsilon_{n}\right)_{n \leq k}$ is a sequence of signs, then the state $s=\left(Z_{n *}, Z_{n}, \varepsilon_{n}\right)_{n \leq k}$ decides $\mathcal{Y}$. 
Such a $\mathcal{Y}$ will be called stabilizing, and states associated to blockings of $\mathcal{Y}$ will be said to be states blocking $\mathcal{Y}$.

Proof. Fix $\mathcal{W}$ in $\operatorname{QS}(X)$. Let $n_{1}$ be such that $\operatorname{dim}\left(W_{n_{1} *}, W_{n_{1}}\right) \geq 1$. We let $\mathcal{Y}^{1}=\mathcal{W}$ and $\left(Y_{1 *}, Y_{1}\right)=\left(\sum_{n \leq n_{1}} Y_{n *}^{1}, \sum_{n \leq n_{1}} Y_{n}^{1}\right)$. Assume $\left(Y_{k *}, Y_{k}\right)_{k<n}$ and some $\mathcal{Y}^{n-1}$ in $\mathrm{QS}_{E}(X)$ have been constructed with $E=\operatorname{ran}\left(\left(Y_{(n-1) *}, Y_{n-1}\right)\right.$. By the finiteness property of $\mathcal{R}$, and the $\leq^{E}$-largeness property of Lemma 14, we may find some $\mathcal{Y}^{n} \leq \mathcal{Y}^{n-1}$, with $\mathcal{Y}^{n} \in \operatorname{QS}_{E}(X)$, such that for any finite sequence $\left(Z_{i *}, Z_{i}\right)_{i \leq m}$ which is a blocking of $\left(Y_{k *}, Y_{k}\right)_{k<n}$ with

$$
\max \left(\operatorname{ran}\left(Z_{m *}, Z_{m}\right)\right)=\max E,
$$

and for any sequence of signs $\left(\varepsilon_{i}\right)_{i \leq m}$, the state $s=\left(Z_{i *}, Z_{i}, \varepsilon_{i}\right)_{i \leq m}$ decides $\mathcal{Y}^{n}$. Let $m_{n}$ be such that $\max \left(\operatorname{ran}\left(Y_{m_{n} *}^{n}, Y_{m_{n}}^{n}\right)=\max E\right.$ and $p_{n}$ be such that the associated subsequence $\left(Y_{i *}^{n}, Y_{i}^{n}\right)_{m_{n}<i \leq p_{n}}$ contains a term of dimension at least 1 . Let $\left(Y_{n *}, Y_{n}\right)$ be $\left(\sum_{m_{n}<i \leq p_{n}} Y_{i *}^{n}, \sum_{m_{n}<i \leq p_{n}} Y_{i}^{n}\right)$.

Repeating this by induction we construct an element $\mathcal{Y}$ of $\mathrm{QS}(X)$ which has the required property. Indeed, for any state $s$ blocking $\mathcal{Y}$, let $n$ be such that $\max (\operatorname{ran}(s))=\max \left(\operatorname{ran}\left(Y_{(n-1) *}, Y_{n-1}\right)\right)$. Then $s$ decides $\mathcal{Y}^{n}$ and $\mathcal{Y} \leq^{s}$ $\mathcal{Y}^{n}$, therefore $s$ decides $\mathcal{Y}$.

We now fix some stabilizing $\mathcal{X}$ in $\mathrm{QS}(X)$. Note that by Lemmas 14 and 15, whenever $s$ is a state blocking $\mathcal{X}$ and $\mathcal{Y} \leq_{s} \mathcal{X}$, then $s$ accepts (resp. rejects) $\mathcal{X}$ if and only if it accepts (resp. rejects) $\mathcal{Y}$. In the following, we shall say that $s$ accepts (resp. rejects) to mean that $s$ accepts (resp. rejects) $\mathcal{X}$.

Lemma 16. Let $s \in S$ be a state blocking $\mathcal{X}$. If s rejects, then for any $\mathcal{Y} \leq \mathcal{X}$ in $\mathrm{QS}_{s}(X)$ there exists $\mathcal{Z} \leq \mathcal{Y}$ in $\mathrm{QS}_{s}(X)$ such that for any block $\left(F_{*}, F\right)$ of $\mathcal{Z}$ which is successive to $s$, and any sign $\varepsilon$, the state $s^{\frown}\left(F_{*}, F, \varepsilon\right)$ rejects.

Proof. Assume the conclusion is false. Let $n=|s|$. There exists $\mathcal{Y} \leq \mathcal{X}$ in $\mathrm{QS}_{s}(X)$ such that for any $\mathcal{Z} \leq \mathcal{Y}$ in $\mathrm{QS}_{s}(X)$, there is a block $\left(F_{(n+1) *}, F_{n+1}\right)$ of $\mathcal{Z}$ successive to $s$ and $\varepsilon_{n+1} \in\{-1,1\}$ such that the state

$$
s^{\prime}=s^{\frown}\left(F_{n+1}^{*}, F_{n+1}, \varepsilon_{n+1}\right)
$$

accepts, and therefore accepts $\mathcal{Y}$, that is, Player 2 has a winning strategy for $G_{\mathcal{A}}^{\mathcal{Y}}\left(s^{\prime}\right)$. Note that $s^{\prime}$ is a state blocking $\mathcal{X}$. What we have written means that Player 2 has a winning strategy for $G_{\mathcal{A}}^{\mathcal{Y}}(s)$, in other words $s$ accepts $\mathcal{Y}$, that is, $s$ accepts. This is a contradiction.

In the following, $\emptyset$ denotes the empty state.

Lemma 17. Assume $\emptyset$ rejects. Then there exists $\mathcal{Y} \leq \mathcal{X}$ such that any state blocking $\mathcal{Y}$ rejects.

Proof. Let $\mathcal{Y}^{0}=\mathcal{X}$. We build by induction a sequence $\mathcal{Y}=\left(Y_{n *}, Y_{n}\right)_{n \in \mathbb{N}}$ and a $\leq$-decreasing sequence $\left(\mathcal{Y}^{n}\right)_{n \in \mathbb{N}}$ with $\mathcal{Y}^{n} \in \operatorname{QS}_{E_{n}}(X)$, with $E_{n}=$ 
$\operatorname{ran}\left(Y_{i *}, Y_{i}\right)_{i<n}$, and with $\left(Y_{n *}, Y_{n}\right)$ a block of $\mathcal{Y}^{n}$ for each $n \in \mathbb{N}$, as follows. Assume $\left(Y_{i *}, Y_{i}\right)_{i<n}$ and $\left(\mathcal{Y}^{i}\right)_{i<n}$ have been defined. There are finitely many states $s$ with $\max (\operatorname{ran}(s))=\max (E)$. Therefore applying Lemma 16 a finite number of times, we obtain some $\mathcal{Y}^{n} \leq \mathcal{Y}^{n-1}$ in $\mathrm{QS}_{E}(X)$ such that for any state $s$ with $\max (\operatorname{ran}(s))=\max (E)$, for any block $\left(F_{*}, F\right)$ of $\mathcal{Y}^{n}$ which is successive to $E$, and for any sign $\varepsilon$, the state $s \frown\left(F_{*}, F, \varepsilon\right)$ rejects. We define $\left(Y_{n *}, Y_{n}\right)$ to be such a block $\left(F_{*}, F\right)$ of dimension at least 1 .

Whenever $\mathcal{U}=\left(U_{n *}, U_{n}\right)_{n \in \mathbb{N}} \leq \mathcal{Y}$, we may easily check by induction that for any sequence of signs $\left(\varepsilon_{i}\right)_{i \leq n}$, the state $\left(U_{i *}, U_{i}, \varepsilon_{i}\right)_{i \leq n}$ rejects.

Proposition 18. Let $\mathcal{A}$ be a subset of $\mathrm{QS}(X) \times\{-1,1\}^{\omega}$ which is open as a subset of $(\mathcal{F}(X) \times\{-1,1\})^{\omega}$ with the product of the discrete topology on $\mathcal{F}(X) \times\{-1,1\}$. If for every $\mathcal{Y} \in \mathrm{QS}(X)$, there exists $\mathcal{Z} \leq \mathcal{Y}$ and a sequence of signs e such that $(\mathcal{Z}, e) \in \mathcal{A}$, then there exists $\mathcal{Y} \in \mathrm{QS}(X)$ such that Player 2 has a winning strategy in the game $G_{\mathcal{A}}^{\mathcal{Y}}$.

Proof. If $\emptyset$ accepts then by definition, Player 2 has a winning strategy in the game $G_{\mathcal{A}}^{\mathcal{Y}}$ for some $\mathcal{Y}$. If $\emptyset$ rejects then, by Lemma 17 , there exists $\mathcal{Y}$ such that any state blocking $\mathcal{Y}$ rejects, which implies that any state blocking $\mathcal{Y}$ is extendable as a sequence which is not in $\mathcal{A}$. Since $\mathcal{A}$ is open, this means that no infinite sequence of successive blocks of $\mathcal{Y}$ and of signs belongs to $\mathcal{A}$.

Recall that for any $\delta>0$, we define $\mathcal{A}_{\delta}$ to be the set of $\left(U_{n *}, U_{n}, \varepsilon_{n}\right)_{n}$ such that there exist $n \in \mathbb{N}$ and $u_{k} \in U_{k}, u_{k}^{*} \in U_{k *}, 1 \leq k \leq n$, such that

$$
\left\|\sum_{k=1}^{n} u_{k}\right\||| \sum_{k=1}^{n} u_{k}^{*} \|<\delta\left|\sum_{k=1}^{n} \varepsilon_{k-1} u_{k}^{*}\left(u_{k}\right)\right|,
$$

where we put $\varepsilon_{0}=1$. This is an open subset of $(\mathcal{F}(X) \times\{-1,1\})^{\omega}$.

2.3. A dichotomy theorem on $\operatorname{QS}(X)$. If $\mathcal{Y} \in \operatorname{QS}(X)$, with $\operatorname{dim}\left(Y_{n *}, Y_{n}\right)$ $=1$ for all $n \in \mathbb{N}$, then we shall write $\mathcal{Y} \in \mathrm{QS}_{1}(X)$. If $\mathcal{Y} \in \mathrm{QS}_{1}(X)$, and for each $n \in \mathbb{N}, \widetilde{e}_{n} \in Y / Y_{*}^{\perp}$ is the class of some $e_{n} \in Y_{n}$ which is not in $Y_{n *}^{\perp}$, then we shall say that $\left(\widetilde{e}_{n}\right)$ is a successive Schauder basis of $Y / Y_{*}^{\perp}$. Note that all successive Schauder bases of $Y / Y_{*}^{\perp}$ may be obtained from one another by homotheties on the span of each of their basic vectors.

In the next proposition, fixing $\delta>0$, we let $\mathcal{X}_{\delta}$ be a stabilizing subspace corresponding to $\mathcal{A}_{\delta}$, and we say that $s \delta$-accepts (resp. $\delta$-rejects) if $s$ accepts (resp. rejects) $\mathcal{X}_{\delta}$ with respect to the set $\mathcal{A}_{\delta}$.

Proposition 19. If $\emptyset \delta$-rejects, then there exists $\mathcal{Y} \in \mathrm{QS}_{1}(X)$ with $\mathcal{Y} \leq \mathcal{X}_{\delta}$ such that any successive basis of $Y / Y_{*}^{\perp}$ is unconditional with constant $\delta^{-1}$. If $\emptyset \delta$-accepts, then whenever $\mathcal{U}, \mathcal{V} \leq \mathcal{X}_{\delta}$ have identical partitions, $A(\mathcal{U}, \mathcal{V})<\delta$.

Proof. If $\emptyset \delta$-rejects, then consider $\mathcal{Y}=\left(Y_{i *}, Y_{i}\right)_{i \in \mathbb{N}}$ given by Lemma 17, and write $E_{i}=\operatorname{ran}\left(Y_{i *}, Y_{i}\right)$. Without loss of generality we may assume that 
$\mathcal{Y} \in \mathrm{QS}_{1}(X)$. Pick in each $Y_{i}$ some normalized $f_{i}$ such that $d\left(f_{i}, Y_{i *}^{\perp} \cap\left[e_{n}\right.\right.$ : $\left.\left.n \in E_{i}\right]\right)=1$. Fix $n$ and some signs $\left(\varepsilon_{i}\right)_{i \leq n}$, and recall that $\varepsilon_{0}=1$. By the proof of Proposition 18, and since $\mathcal{A}_{\delta}$ is open, for any $\left(y_{i}^{*}, y_{i}\right) \in Y_{i *} \times Y_{i}$, $i \leq n$, we have

$$
\left\|\sum_{k=1}^{n} y_{k}\right\||| \sum_{k=1}^{n} y_{k}^{*} \| \geq \delta\left|\sum_{k=1}^{n} \varepsilon_{k-1} y_{k}^{*}\left(y_{k}\right)\right| .
$$

Equivalently, whenever $\left\|\sum_{k=1}^{n} y_{k}^{*}\right\|=1$,

$$
\left(\sum_{k=1}^{n} y_{k}^{*}\right)\left(\sum_{k=1}^{n} \varepsilon_{k-1} y_{k}\right) \leq \delta^{-1}\left\|\sum_{k=1}^{n} y_{k}\right\|,
$$

and therefore

$$
\left\|\sum_{k=1}^{n} \varepsilon_{k-1} y_{k}\right\|_{\sum_{k \leq n} Y_{k *}} \leq \delta^{-1}\left\|\sum_{k=1}^{n} y_{k}\right\| .
$$

Taking $y_{k}=\lambda_{k} f_{k}+z_{k}$, where $\lambda_{k}$ is a scalar and $z_{k}$ is arbitrary in $Y_{k *}^{\perp} \cap\left[e_{i}\right.$ : $i \in E_{k}$ ], we obtain

$$
\left\|\sum_{k=1}^{n} \varepsilon_{k-1} \lambda_{k} f_{k}\right\|_{\sum_{k \leq n} Y_{k *}} \leq \delta^{-1}\left\|\sum_{k=1}^{n} \lambda_{k} f_{k}+z\right\|,
$$

where $z \in \sum_{k \leq n}\left(Y_{k *}^{\perp} \cap\left[e_{i}: i \in E_{k}\right]\right)=\left(\sum_{k \leq n} Y_{k *}\right)^{\perp} \cap\left[e_{i}: i \in \bigcup_{k \leq n} E_{k}\right]$ is arbitrary. By duality in $\left[e_{i}: i \in \bigcup_{k \leq n} E_{k}\right]$, we conclude that

$$
\left\|\sum_{k=1}^{n} \varepsilon_{k-1} \lambda_{k} f_{k}\right\|_{\sum_{k \leq n} Y_{k *}} \leq \delta^{-1}\left\|\sum_{k=1}^{n} \lambda_{k} f_{k}\right\|_{\sum_{k=1}^{n} Y_{k *}} .
$$

Since $\left(\varepsilon_{i}\right)_{1 \leq i \leq n-1}$ was arbitrary, we deduce that $\left(\widetilde{f}_{k}\right)_{k \leq n}$ is $\delta^{-1}$-unconditional in $\sum_{k \leq n} Y_{k} /\left(\left(\sum_{k \leq n} Y_{k *}\right)^{\perp} \cap\left[e_{i}: i \in \bigcup_{k \leq n} E_{k}\right]\right)$ for each $n$, and therefore in $Y / Y_{*}^{\perp}$ by bimonotonicity.

Now assume $\emptyset \delta$-accepts. Pick $\mathcal{U}, \mathcal{V} \leq \mathcal{X}_{\delta}$ which have identical partitions. This will ensure that playing $\mathcal{U}$ or $\mathcal{V}$ is always an admissible move for Player 1 . We therefore may define a strategy for Player 1 as follows. The first move is $\mathcal{U}$. Assuming Player 2 has picked some $\left(Y_{k-1}^{*}, Y_{k-1}, \varepsilon_{k-1}\right)$ at step $k-1$, Player 1's $k$ th move will be $\mathcal{U}$ if $\varepsilon_{k-1}=1$, and $\mathcal{V}$ if $\varepsilon_{k-1}=-1$. Opposing a winning strategy for Player 2 , we therefore obtain some $n \in \mathbb{N}$, and some sequences $\left(u_{i}^{*}, u_{i}\right)_{i \leq n}$ of pairs of vectors and functionals, and $\left(\varepsilon_{i}\right)_{i \leq n}$ of signs such that $u_{i} \in U, u_{i}^{*} \in U_{*}$ if $\varepsilon_{i-1}=1$, and $u_{i} \in V, u_{i}^{*} \in V_{*}$ if $\varepsilon_{i-1}=-1$, and with

$$
\left\|\sum_{k=1}^{n} u_{k}\right\||| \sum_{k=1}^{n} u_{k}^{*} \|<\delta\left|\sum_{k=1}^{n} \varepsilon_{k-1} u_{k}^{*}\left(u_{k}\right)\right| .
$$

We let $u=\sum_{\varepsilon_{i-1}=1} u_{i} \in U, u^{*}=\sum_{\varepsilon_{i-1}=1} u_{i}^{*} \in U_{*}, v=-\sum_{\varepsilon_{i-1}=-1} u_{i} \in V$, 
$v^{*}=-\sum_{\varepsilon_{i-1}=-1} u_{i}^{*} \in V_{*}$, and observe that $u^{*}(v)=v^{*}(u)=0$ and

$$
\|u-v\|\left\|u^{*}-v^{*}\right\|<\delta\left|u^{*}(u)-v^{*}(v)\right| \text {. }
$$

Theorem 20. Let $X$ be a Banach space with a Schauder basis. Then there exists a quotient $Y / Y_{*}^{\perp}$ of a subspace of $X$, associated to some $\mathcal{Y}$ in $\operatorname{QS}_{1}(X)$, which has one of the following two properties, which are both possible and mutually exclusive:

(i) $Y / Y_{*}^{\perp}$ has an unconditional basis,

(ii) $Y / Y_{*}^{\perp}$ has the restricted QHI property.

Proof. Fix as before a positive sequence $\left(\delta_{n}\right)_{n \in \mathbb{N}}$ with $\delta_{n} \leq 2^{-n}$ for all $n$, and build by Lemma 15 a $\leq$-decreasing sequence $\mathcal{X}_{n}$ such that $\mathcal{X}_{n}$ is $\delta_{n^{-}}$ stabilizing for each $n$. If, in the terminology defined at the beginning of this subsection, $\emptyset \delta_{n}$-rejects $\mathcal{X}_{n}$ for some $n$, then we are done by Proposition 19.

Assume therefore that $\emptyset \delta_{n}$-accepts $\mathcal{X}_{n}$ for all $n \in \mathbb{N}$. Let $\mathcal{Y} \in \operatorname{QS}(X)$ be diagonal for the $\mathcal{X}_{n}$ 's, i.e. such that $\mathcal{Y} \leq^{s} \mathcal{X}_{n}$ for any state $s$ blocking $\mathcal{Y}$ with $\max (\operatorname{ran}(s))=\max \left(\operatorname{ran}\left(Y_{n *}, Y_{n}\right)\right)$. This is easily constructed by induction. We shall prove that $A(\mathcal{U}, \mathcal{V})=0$ for any $\mathcal{U}, \mathcal{V} \leq \mathcal{Y}$ which are sequences of successive blocks (not necessarily in $\mathcal{R}$ ) with equal ranges forming a partition of $\mathbb{N}$. By Lemma 8 , this will be enough to prove our result.

Fix $\varepsilon>0$ and arbitrary $\mathcal{U}, \mathcal{V} \in \mathrm{QS}(X)$ (therefore formed of $\mathcal{R}$-blocks), with $\mathcal{U}, \mathcal{V} \leq \mathcal{Y}$ and with identical partition denoted $\left(E_{n}\right)_{n \in \mathbb{N}}$. Let $m$ be large enough so that if $p=\max \left(E_{m}\right)$ then $\delta_{p}<\varepsilon$. Denote by $\mathcal{X}=\left(X_{i *}, X_{i}\right)_{i \in \mathbb{N}}$ the corresponding $\mathcal{X}_{p}$, by $F_{i}$ the range of $\left(X_{i *}, X_{i}\right)$ and let $q$ be such that $p=\max \left(F_{q}\right)$.

For $i \leq q$ we let $U_{i *}^{\prime}=X_{i *}$, and $U_{i}^{\prime}=X_{i *}^{\perp} \cap\left[e_{n}: n \in F_{i}\right]$. For $i>q$ we let $\left(U_{i *}^{\prime}, U_{i}^{\prime}\right)=\left(U_{(m-q+i) *}, U_{m-q+i}\right)$. We have thus constructed an element $\mathcal{U}^{\prime}$ of $\mathrm{QS}(X)$ which satisfies $\mathcal{U}^{\prime} \leq \mathcal{X}$ and $\mathcal{U}^{\prime} \leq^{E} \mathcal{U}$ for $E=[1, p]$. In the same way we construct some $\mathcal{V}^{\prime} \leq \overline{\mathcal{X}}$ with $\mathcal{V}^{\prime} \leq^{E} \mathcal{V}$.

By Proposition 19, we may find $x \in U^{\prime}, x^{*} \in U_{*}^{\prime}$ and $y \in V^{\prime}, y^{*} \in V_{*}^{\prime}$ with disjoint supports and with $\|x-y\|\left\|x^{*}-y^{*}\right\|<\delta_{p}\left|\left(x^{*}-y^{*}\right)(x+y)\right|$. Let $P$ be the projection onto $\left[e_{n}: n>p\right]$, and $P_{*}$ be the projection onto $\left[e_{n}^{*}: n>p\right]$. Note that $P\left(U^{\prime}\right) \subset U$ and $P_{*}\left(U_{*}^{\prime}\right) \subset U_{*}$, and similar inclusions hold for $V$ and $V_{*}$. Let $u=P x, u^{*}=P_{*} x^{*}, v=P y, v^{*}=P_{*} y^{*}$.

By bimonotonicity of the basis, we observe that $\|u-v\| \leq\|x-y\|$ and $\left\|u^{*}-v^{*}\right\| \leq\left\|x^{*}-y^{*}\right\|$. On the other hand, writing $x=u+a, x^{*}=u^{*}+a^{*}$, $y=v+b$, and $y^{*}=v^{*}+b^{*}$, we note that $a \in\left(\sum_{i \leq q} X_{i *}\right)^{\perp}$ while $a^{*} \in$ $\sum_{i \leq q} X_{i *}$, therefore $a^{*}(a)=0$. Likewise, $b^{*}(b)=a^{*}(b) \stackrel{\leq q}{=} b^{*}(a)=0$, and by disjointness of the ranges, $u^{*}(a)=u^{*}(b)=v^{*}(a)=v^{*}(b)=a^{*}(u)=a^{*}(v)=$ $b^{*}(u)=b^{*}(v)=0$. Therefore

$$
\left(x^{*}-y^{*}\right)(x+y)=\left(u^{*}-v^{*}\right)(u+v),
$$


and we deduce that

$$
\|u-v\|\left\|u^{*}-v^{*}\right\|<\delta_{p}\left|\left(u^{*}-v^{*}\right)(u+v)\right| .
$$

We have thus proved that $A(\mathcal{U}, \mathcal{V})<\varepsilon$.

It remains to show that we may obtain the same results for general $\mathcal{U}, \mathcal{V} \leq \mathcal{Y}$, i.e. successive sequences of blocks which are not necessarily in $\mathcal{R}$. Fix $0<\varepsilon<1 / 3$ and let $\mathcal{U}, \mathcal{V}$ have the same partition $\left(E_{n}\right)_{n \in \mathbb{N}}$. Let $\mathcal{U}^{\prime}, \mathcal{V}^{\prime}$ be sequences with blocks in $\mathcal{R}$ such that $\delta\left(\left(U_{n *}, U_{n}\right),\left(U_{n *}^{\prime}, U_{n}^{\prime}\right)\right)<\delta_{n}$ for all $n \in \mathbb{N}$, and with similar relations for $\left(V_{n *}, V_{n}\right)$ and $\left(V_{n *}^{\prime}, V_{n}^{\prime}\right)$. Let $N \in \mathbb{N}$ be such that $2^{-N} \leq \varepsilon / 2$. By the above, we may find a partition $\{I, J\}$ of $[N, \infty)$, vectors $u \in \sum_{n \in I} U_{n}^{\prime}, v \in \sum_{n \in J} V_{n}^{\prime}$, and functionals $u^{*} \in \sum_{n \in I} U_{n *}^{\prime}$, $v^{*} \in \sum_{n \in J} V_{n *}^{\prime}$ such that

$$
\|u-v\|\left\|u^{*}-v^{*}\right\|<\varepsilon\left|\left(u^{*}-v^{*}\right)(u+v)\right| .
$$

For $n \geq N$, let $\left(W_{n *}, W_{n}\right)=\left(U_{n *}, U_{n}\right)$ if $n \in I$, and $\left(W_{n *}, W_{n}\right)=$ $\left(V_{n *}, V_{n}\right)$ if $n \in J$, and let $W_{*}=\sum_{n \geq N} W_{n *}$; let $\left(W_{n *}^{\prime}, W_{n}^{\prime}\right)$ and $W_{*}^{\prime}$ be defined in a similar way. Let also $X_{N}=\left[e_{i}: i \in \bigcup_{n \geq N} E_{n}\right]$. We then have

$$
\|u-v\|<\varepsilon\|u+v\|_{W_{*}^{\prime}} .
$$

Since $\sum_{n \geq N} \delta_{n} \leq \varepsilon$, we find by Lemma 9 a map $T$ from $\sum_{n \geq N} W_{n}^{\prime}$ onto $\sum_{n \geq N} W_{n}$ such that $T\left(W^{\prime}\right)=W, T\left(X_{N} \cap W_{*}^{\prime \perp}\right)=X_{N} \cap W_{*}^{\perp}$, and

$$
\|T\|\left\|T^{-1}\right\| \leq(1+\varepsilon)(1-\varepsilon)^{-1} \leq 2 .
$$

If we let $x=T u \in \sum_{n \in I} U_{n}$ and $y=T v \in \sum_{n \in J} V_{n}$, we have

$$
\|x-y\|<2 \varepsilon\|x+y\|_{W_{*}} \text {. }
$$

This means that we may pick some normalized functional $w^{*} \in W_{*}$, hence $w^{*}=x^{*}-y^{*}$ with $x^{*} \in \sum_{n \in I} U_{n *}$ and $y^{*} \in \sum_{n \in J} V_{n *}$, such that

$$
|| x-y \|<2 \varepsilon\left|\left(x^{*}-y^{*}\right)(x+y)\right|=2 \varepsilon\left|x^{*}(x)-y^{*}(y)\right|,
$$

and we deduce that $A(\mathcal{U}, \mathcal{V}) \leq 2 \varepsilon$.

\section{Remarks and open questions}

REMARK 21. Let $Y$ be an FDD-block quotient of a subspace of $X$. To check whether $Y$ has the restricted QHI property, we have checked the formally stronger result that the angle is zero between any two QS-pairs associated to FDD-block quotients of subspaces of $Y$ with sequences of blocks having the same partition. We note that by our dichotomy theorem, these two notions are equivalent up to passing to a quotient of a subspace. Indeed, if $X$ is QHI restricted to block-subspaces, then no quotient of $X$ by an FDD-block subspace can contain an unconditional basic sequence, and therefore $X$ must contain a quotient of a subspace with the stronger "angle zero" property. 
Question 22. Is it possible to improve Theorem 20 by suppressing the restriction to FDD-block subspaces? This restriction is not only technical. By a result of S. Argyros, A. Arvanitakis and A. Tolias [1], the distinction between general quotient spaces and quotients by FDD-block subspaces may be essential: there exists a separable dual space $X$ with a Schauder basis such that quotients with $w^{*}$-closed kernels are HI, yet every quotient has a further quotient isomorphic to $l_{2}$. Since FDD-block subspaces of $X$ are $w^{*}$-closed, this space has the restricted QHI property, but it is not QHI by the $l_{2}$-saturation property.

Unlike the case of subspaces, it does not seem clear that, in a space with a Schauder basis, QS-spaces may be approximated by FDD-block quotients of subspaces, that is, we do not know whether for any QS-space, there is a further QS-space which is an arbitrarily small perturbation of an FDD-block quotient of a subspace.

REMARK 23. As noticed in the introduction, HI spaces can fall in either side of the dichotomy in Theorem 6. The example of $X_{\mathrm{GM}}$ is QHI, while the examples of [2] have an unconditional quotient. The dual $X_{\mathrm{uh}}^{*}$ of the reflexive space $X_{\text {uh }}$ of Argyros and Tolias [5] has the following quite interesting mixed property. Any of its quotients has a further quotient with an unconditional basis [5, Proposition 3.6], but on the other hand it is HI [5, Proposition 5.11], and it is saturated with QHI subspaces. This last fact was indicated to us by S. Argyros, and the proof is as follows. Consider any block subspace of $X_{\mathrm{uh}}^{*}$. Keeping half of the vectors of the block basis, and denoting by $Y$ the space generated by them, we find that the annihilator of any subspace $Z$ of $Y$ must contain an infinite subsequence of the basis. Therefore [5, Proposition 6.3] applies to show that $X_{\mathrm{uh}} / Z^{\perp}$ is HI. This means that every infinite-dimensional quotient of $X_{\mathrm{uh}} / Y^{\perp}$ is HI, therefore $X_{\mathrm{uh}} / Y^{\perp}$ is QHI. By reflexivity it follows that $Y \simeq\left(X_{\mathrm{uh}} / Y^{\perp}\right)^{*}$ is QHI.

We say that a Banach space $X$ is unconditionally QS-saturated (resp. $Q S$-saturated with HI subspaces) if any infinite-dimensional QS-space of $X$ has a further QS-space with an unconditional basis (resp. which is HI).

By Odell's result [13], if a space $X$ has a shrinking unconditional FDD, then every quotient of $X$ must be unconditionally saturated, and therefore $X$ must be unconditionally QS-saturated. It remains unknown whether there exists a HI space which is unconditionally QS-saturated. Therefore we ask:

Does every HI space contain a quotient of a subspace which is QHI? or which has the restricted QHI property?

REMARK 24. Our dichotomy theorem, the result of Odell [13], and the remark after Proposition 5 imply the following: if $X$ is reflexive and QS- 
saturated with HI spaces, then some quotient of a subspace of $X$ has the restricted QHI property, and is therefore $\mathrm{HI}$ and saturated with subspaces with HI dual.

In this direction, we recall the question of S. Argyros: does there exist a reflexive HI space $X$ such that no subspace of $X$ has a HI dual?

\section{References}

[1] S. Argyros, A. Arvanitakis and A. Tolias, Saturated extensions, the attractors method, and hereditarily James tree spaces, in: Methods in Banach Space Theory, London Math. Soc. Lecture Note Ser. 337, Cambridge Univ. Press, 2006, 1-90.

[2] S. Argyros and V. Felouzis, Interpolating hereditarily indecomposable Banach spaces, J. Amer. Math. Soc. 13 (2000), 243-294.

[3] S. Argyros and S. Todorcevic, Ramsey Methods in Analysis, Adv. Courses Math. CRM Barcelona, Birkhäuser, Basel, 2005.

[4] S. Argyros and A. Tolias, Methods in the theory of hereditarily indecomposable Banach spaces, Mem. Amer. Math. Soc. 170 (2004), no. 806.

[5] -, - Indecomposability and unconditionality in duality, Geom. Funct. Anal. 14 (2004), 247-282.

[6] V. Ferenczi, Quotient hereditarily indecomposable Banach spaces, Canad. J. Math. 51 (1999), 566-584.

[7] -, Topological 0-1 laws for subspaces of a Banach space with a Schauder basis, Illinois J. Math. 49 (2005), 839-856.

[8] W. T. Gowers, An infinite Ramsey theorem and some Banach-space dichotomies, Ann. of Math. (2) 156 (2002), 797-833.

[9] W. T. Gowers and B. Maurey, The unconditional basic sequence problem, J. Amer. Math. Soc. 6 (1993), 851-874.

[10] J. Lindenstrauss and L. Tzafriri, Classical Banach Spaces, Springer, New York, 1979.

[11] B. Maurey, Banach spaces with few operators, in: Handbook of the Geometry of Banach Spaces, Vol. 2, W. B. Johnson and J. Lindenstrauss (eds.), Elsevier, Amsterdam, 2003, 1247-1297.

[12] —, Quelques progrès dans la compréhension de la dimension infinie, in: Espaces de Banach classiques et quantiques, SMF Journ. Ann., Soc. Math. France, 1994, 29 pp.

[13] T. Odell, On quotients of Banach spaces having shrinking unconditional bases, Illinois J. Math. 36 (1992), 681-695.

Institut de Mathématiques de Jussieu

Projet Analyse Fonctionnelle

Université Pierre et Marie Curie - Paris 6

Boîte 186, 4, Place Jussieu

75252 Paris Cedex 05, France

E-mail: ferenczi@ccr.jussieu.fr

Received March 1, 2006

Revised version March 6, 2007 